\title{
THE MEASUREMENT OF INTERFACE STATE CHARGE IN THE MOS SYSTEM
}

\author{
JAN KOOMEN \\ Twente University of Technology, Enschede, Netherlands
}

(Received 16 September 1970)

\begin{abstract}
A simple method of measuring charge in surface states as a function of the surfacepotential in MOS transistors or MOS capacitors is proposed.

A constant d.c. current is fed into the gate of an MOS transistor and with the help of an operational amplifier, the gate voltage $V_{g}$ with respect to the bulk is plotted as a function of gate charge $Q_{g}$. The gate charge as a function of the surfacepotential $\phi_{s}$ can be directly read off from the $V_{g}-Q_{g}$ curve.

As the silicon charge $Q_{s}\left(\phi_{s}\right)$ is known from the literature the surface state charge can be easily determined as a function of $\phi_{s}$. The method is illustrated with measurements on $n$-type and $p$-type MOS transistors. Finally the accuracy of the charge measuring method is discussed and a comparison with other interface state charge measuring methods is made from which the charge measuring method evolves as a method, attractive for its simplicity.
\end{abstract}

Résumé - Une méthode simple pour mesurer la charge d'états de surface en fonction du potentiel de surface $\phi_{s}$ dans des transistors MOS ou des condensateurs MOS est proposée.

On charge à courant constant la grille d'un transistor MOS et à l'aide d'un amplificateur opérationnel la tension grille-substrat $V_{g}$ est registrée en fonction de la charge de grille $Q_{g}$. De la courbe $V_{g}-Q_{g}$ il est possible de déterminer directement la caractéristique $\phi_{s}-Q_{\sigma}$.

Parce que la charge dans l'intérieur du silicium sous l'oxyde de grille $Q_{s}\left(\phi_{g}\right)$ est connue de la littérature on peut déterminer facilement la charge d'états de surface en fonction de $\phi_{s}$. La méthode est appliquée aux quelques transistors MOS à substrat $n$ et $p$. Aussi on discute la précision de la méthode proposée et on fait une comparaison avec des méthodes existantes. De cette comparaison on peut concluire que la méthode proposée est attractive par sa simplicité.

Zusammenfassung - Eine einfache Methode zur Ladungsmessung in Oberflächenzuständen als $\Gamma$ unktion des Oberflächenpotentials in MOS Transistoren und MOS Kondensatoren wird vorgeschlagen.

Ein konstanter Gleichstrom wird der Steuerelektrode eines MOS Transistors zugeführt. Mittels eines Operationsverstärkers wird die Steuerspannung $V_{g}$ bezogen auf den Substrat, als Funktion der Steuerelektrodenladung $Q_{g}$ geschrieben. Die Steuerelektrodenladung als Funktion des Oberflächenpotentials $\phi_{s}$ kann direkt aus der $V_{g}-Q_{g}$ Kennlinie abgelesen werden.

Da die Siliziumladung $Q_{s}\left(\phi_{s}\right)$ aus der Literatur bekannt ist, kann die Oberflächenzustandsladung leicht als Funktion von $\phi_{s}$ bestimmt werden. Die Methode wird durch Messungen an MOS Transistoren vom $n$ - und $p$-Typ verdeutlicht. Schlieszlich wird die Genauigkeit der Ladungsmessungsmethode diskutiert und mit anderen Methoden zur Bestimmung der Oberflächenladung verglichen. Hieraus ergibt sich als Vorzug der Ladungsmessungsmethode, ihre grosze Einfachheit.

\section{INTRODUCTION}

The DETERMINATION of the charge distribution at the $\mathrm{Si}-\mathrm{SiO}_{2}$ interface in MOS capacitors or transistors has been the subject of a number of papers [1-14]. Knowledge of the charge distribution, and more precisely, knowledge about the charge in the so-called 'interface states' is of value for those who are concerned about the electrical performance of MOS capacitors and transistors.

The method most employed for a determination of the charge distribution is based upon a differential capacitance measurement on the MOS system under varying d.c. bias. This method, as well as its interpretation, has been discussed extensively [2-9]. In this paper we intend to present an alternative charge measuring method, which is based upon the direct measurement of charge supplied to the MOS system as a function of bias. One of the advantages of the method is its physical clearness. 


\section{THE CHARGE MEASURING METHOD}

In Fig. 1(a) we have depicted a parallel plate condenser, being charged by a current $l_{0}$. When we plot (Fig. 1(b)) the capacitor voltage $V_{g}$ vs. the plate charge $Q_{y}$, we find a straight line with slope $C_{0}{ }^{-1}$ if $C_{0}$ is the capacitance of the condenser. In the case that the two metal plates are similar and their work functions equal, the straight line in Fig. 1(b) will pass through the origin. However, when the work function difference between top plate and bottom plate $\Phi_{1}-\Phi_{2}=\Phi_{12}$ is finite, the plate charge will just be zero when $V_{g}$ is equal to the contact potential $\phi_{12}=-q^{-1} \Phi_{12}$. In general the capacitor voltage $V_{g}$ satisfies:

$$
V_{g}=\phi_{12}+Q_{g} \cdot C_{0}
$$

We take a step further by assuming that the bottom plate of the condenser in Fig. 1(a) is replaced by a piece of silicon. The top plate is equivalent to the 'gate' and between gate and silicon a dielectric medium like $\mathrm{SiO}_{2}$ is present. Now we must remember that, unlike the case for the metal plate condenser a potential will develop in the silicon over a finite distance, if surface charge is applied. If $\phi_{s}$ is the potential at the silicon surface with respect to its interior, we can write down the following relationship between gate voltage $V_{g}$ and gate charge $Q_{g}$ :

$$
V_{g}=\phi_{M S}+Q_{g} \cdot C_{0}^{-1}+\phi_{s}
$$

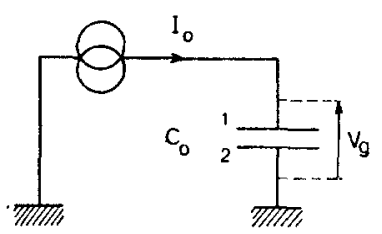

Fig. 1(a). A parallel plate condenser, charged by a constant d.c. current.

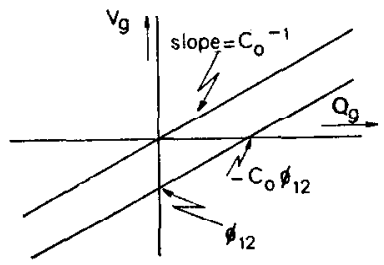

Fig. 1(b). The voltage $V_{s}$ as a function of the plate charge $Q_{g}$ across the capacitor $C_{0}$ in Fig. 1(a).
$\phi_{M S}$ denotes the contact potential between gate metal and silicon. Equation (2) expresses that for the MOS system $V_{g}$ as a function of $Q_{g}$ behaves very much like in the case of a parallel metal plate condenser, except that a displacement $\phi_{\mathrm{s}}$ along the $V_{g}$ axis, which is dependent on $Q_{g}$, has to be accounted for.

Under the condition of strong surface accumulation $\left(V_{g} \gg 0\right.$ for $n$-type and $V_{g} \ll 0$ for $p$-type silicon) or strong surface inversion $\left(V_{g} \ll 0\right.$ for $n$-type and $V_{g} \gg 0$ for $p$-type silicon), $\phi_{\mathrm{s}}$ attains a more or less constant value and the slope of $V_{g} \mathrm{vs}$. $Q_{y}$ tends to $C_{0}{ }^{-1}$ (Fig. 2).

There is an intermediate region, where $\phi_{s}$ changes from its minimum to its maximum value.

Assuming that the value of the surfacepotential $\phi_{s}$ in point $A$ is known (Fig. 2) lines of equal and known surfacepotential can be drawn in the measured $V_{g}-Q_{g}$ plot. According to expression (2) all these lines should have a slope $C_{0}{ }^{-1}$. From the intersections of the equi-surfacepotential lines with the $V_{g}-Q_{g}$ plots, $Q_{g}$ and $V_{g}$ can be determined as a function of $\phi_{s}$.

We now have a closer look at $Q_{g}$, the plate charge. For reasons of neutrality $Q_{g}$ should be compensated by a charge $-Q_{g}$ on the silicon side, which consists of four components:

- depletion charge of ionized bulk impurities

- excess charge carriers in the silicon, near the interface

- charge $Q_{s s}$ in interface states, which interacts with charge carriers in the silicon

- constant charge $Q_{0}$ in the dielectric medium, which is assumed to be located near the interface.

When the charge inside the silicon-the sum of the two first contributions - is denoted by $Q_{s}$, we may write:

$$
Q_{o}=-\left(Q_{s}+Q_{s s}+Q_{0}\right)
$$

Provided that the silicon doping level is known and conditions of thermal equilibrium exist, the silicon charge $Q_{s}$ is known as a function of $\phi_{s}[1,15]$. Substitution of $Q_{g}\left(\phi_{s}\right)$ and $Q_{s}\left(\phi_{s}\right)$ into (3) yields $Q_{s s}+Q_{0}$ as a function of the surfacepotential $\phi_{s}$, which is the main objective of our paper.

So far we have indicated how, in principle, from the measurement of gate voltage vs. gate charge on an MOS system, information can be obtained about the interface state charge density as a function of surfacepotential. 
Before we proceed to a description of the actual measurement set-up and the charge measurements, we have still to explain in detail how essential parameters like oxide capacitance $C_{0}$ and the surfacepotential $\phi_{s}$ are determined and how they can be used for a detailed interpretation of measured $V_{g}-Q_{g}$ plots.

\section{THE EVALUATION OF GATE VOLTAGE VS. GATE CHARGE PLOTS}

This section treats the determination of the surface potential $\phi_{s}$ as a function of $V_{g}$ from an analysis of the $V_{g}$ vs. $Q_{g}$ plot.

In the condition of very strong accumulation or very strong inversion the silicon charge will be dominated by excess charge carriers near the interface and equation [3] reduces to $Q_{g} \approx-Q_{s}$. Theoretical considerations $[1,15]$ lead to the following expressions for the silicon charge:

$$
\begin{aligned}
& \text { bulk: } p \text {-type } \\
& \text { inversion }\left(\phi_{s} \gg 0\right) \\
& Q_{s}=-q L n_{i}\left(\frac{n_{i}}{C_{B}}\right)^{1 / 2} \exp \left(\frac{q \phi_{s}}{2 k T}\right) \\
& \text { bulk: } n \text {-type } \\
& \text { inversion }\left(\phi_{s} \ll 0\right) \\
& Q_{s}=+q L n_{i}\left(\frac{n_{i}}{C_{B}}\right)^{1 / 2} \exp \left(\frac{-q \phi_{s}}{2 k T}\right)
\end{aligned}
$$

In these expressions $n_{i}$ is the intrinsic concentration $\left(1.5 \times 10^{10} \mathrm{~cm}^{-3} ; 300^{\circ} \mathrm{K}\right) ; L$ is the DebyeHückel length: $\left(2 \epsilon_{s i} k T / q n_{i}\right)^{1 / 2}=4.8 \times 10^{-3} \mathrm{~cm}$ at $300^{\circ} \mathrm{K} ;(k T / q)=26 \cdot 10^{-3} \mathrm{~V}$ at $300^{\circ} \mathrm{K}$; $\epsilon_{s i}$ is the permittivity of silicon and $C_{B}$ is the bulk dope concentration.

In Fig. 3(a) $V_{g}$ vs. $Q_{g}$ plot, measured on an $n$-type bulk MOS transistor is shown. When we consider two points $A$ and $B$ on this plot equally far into inversion and accumulation and we assume that for these points $Q_{D}=-Q_{s}$ is given by expressions (4) and furthermore that $\left|Q_{s, A}\right|=\left|Q_{s, B}\right|$ then we can derive:

$$
\begin{aligned}
\left|\phi_{s, A}-\phi_{s, B}\right| & =\frac{4 k T}{q} \ln \frac{\left|Q_{s, A \text { or } B}\right|}{q L n_{i}} \\
\frac{\phi_{s, A}+\phi_{s, B}}{2} & =-\frac{k T}{q} \ln \frac{C_{B}}{n_{i}}
\end{aligned}
$$

Equation (5) yields the range of $\phi_{s}$ values covered by the $V_{g}-Q_{o}$ measurement between $A$ and $B$. It is apparent from (5) that this range is independent of the bulk dope $C_{B}$. Figure 4 shows a plot of computed $\left|\phi_{s, A}-\phi_{s, H}\right|$ values as a function of $Q_{s, A \text { or } B}$. Equation (6) indicates that the average surfacepotential is the potential for which the surface becomes intrinsic. The average surfacepotential as a function of dope concentration $C_{B}$ is shown in Fig. 5.

With the aid of the above knowledge we can again analyse the $V_{g}-Q_{g}$ plot. According to expression (2) in point $A$ of Fig. 3:

$$
V_{g, A}=\phi_{M S}+Q_{g, A} \cdot C_{0}^{-1}+\phi_{s, A}
$$

We can now obtain from Fig. 4 the surfacepotential interval $\left|\phi_{s, A}-\phi_{s, B}\right|$ associated with the charge $\left|Q_{s, A}\right|$. When in point $A$ a vertical line segment of

$\left.\begin{array}{l}\text { accumulation }\left(\phi_{s} \ll 0\right) \\ Q_{s}=+q L n_{i}\left(\frac{C_{B}}{n_{i}}\right)^{1 / 2} \exp \left(\frac{-q \phi_{s}}{2 k T}\right) \\ \text { accumulation }\left(\phi_{s} \gg 0\right) \\ Q_{s}=-q L n_{i}\left(\frac{C_{B}}{n_{i}}\right)^{1 / 2} \exp \left(\frac{q \phi_{s}}{2 k T}\right)\end{array}\right\}$

length $\left|\phi_{s, A}-\phi_{s, B}\right|$ is drawn, the top $A^{\prime}$ of this segment will represent a gate voltage:

$$
V_{g, A^{\prime}}=\phi_{M S}+Q_{g \cdot A} \cdot C_{0}^{-1}+\phi_{s, B}
$$

In point $B$ the gate voltage obeys:

$$
V_{g, B}=\phi_{M S}+Q_{g, B} \cdot C_{0}^{-1}+\psi_{s, B}
$$

or by subtraction we find:

$$
C_{0}=\frac{Q_{g_{B} B}-Q_{g, A}}{V_{g, B}-V_{g, A^{\prime}}}
$$

The construction of Fig. 3, based on expression (7) allows us to determine $C_{0}$. It also furnishes the means to construct the equi-surfacepotential lines, as we have described in the preceeding sections by drawing straight lines parallel to $A^{\prime} / B$. It should 


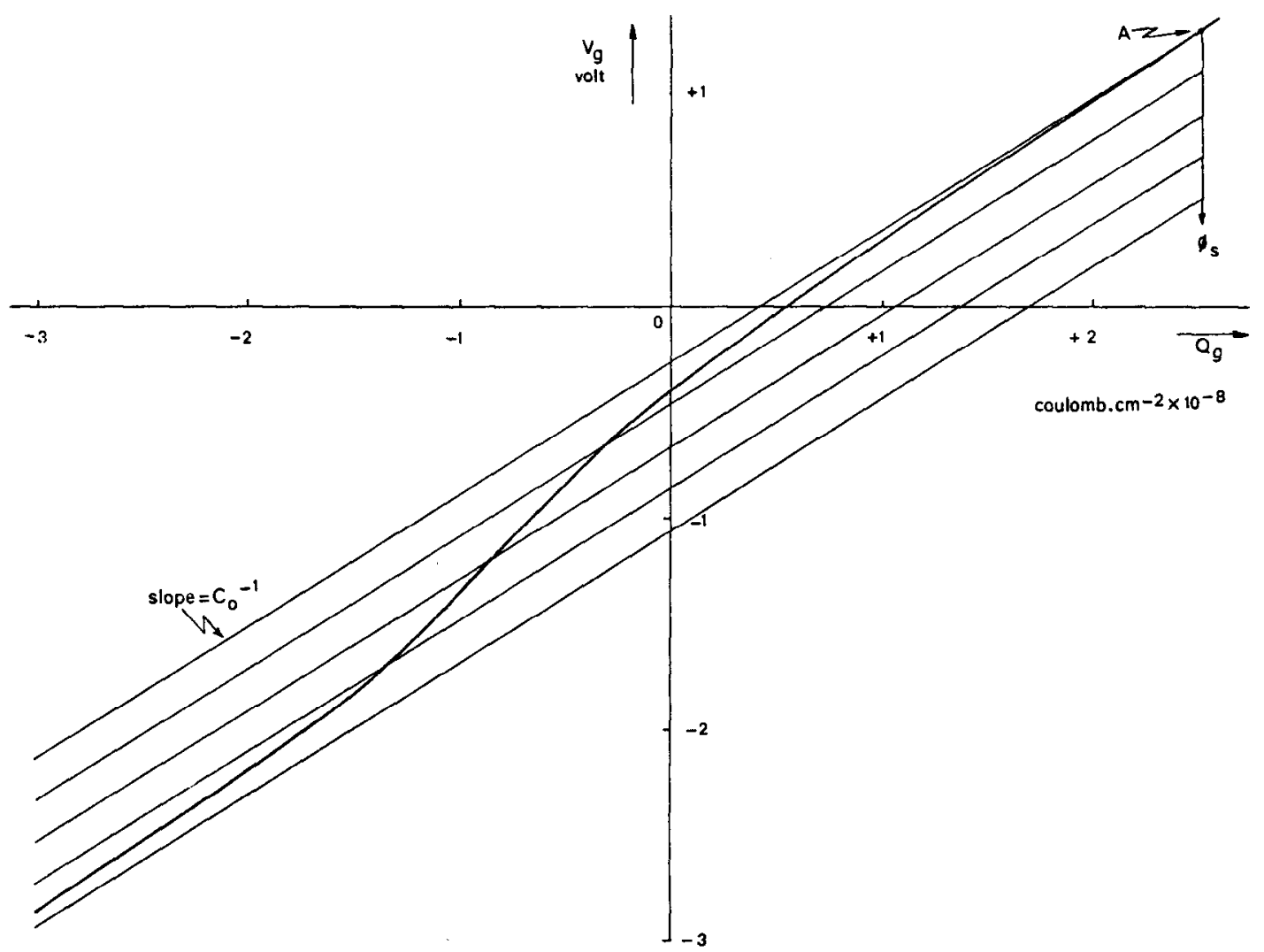

Fig. 2. The gate voltage $V_{g}$ as a function of the gate charge $Q_{g}$ for an $n$-type MOS transistor (sample 2 , listed in Table 1).

be clear that due to expression (6) the equisurfacepotential line which crosses $A A^{\prime}$ half way is the 'intrinsic' surface line. When the potential associated with this line is known from an evaluation of (6), the potentials associated with all other lines are also known.

An equi-surfacepotential line of special importance is the line $\phi_{s}=0$ which according to (2) crosses the $V_{g}$ axis in $V_{g}=\phi_{M S}$ (Fig. 6). The intersection between the line $\phi_{s}=0$ and the $V_{g}$ $Q_{g}$ plot indicates the 'flat-band' situation where $Q_{s}=0$.

A prerequisite for the derivation of the surface potential $\phi_{s}$ of the equi-surfacepotential lines is the existence of two points $A$ and $B$ on the $V_{g}-Q_{g}$ plot, equally far into inversion and accumulation, where the silicon charge should be dominated by charge carriers and the two values of the charges should be equal. Inevitably the determination of the location of $A$ and $B$ will be subject to inaccuracy.
Errors in the estimate of $Q_{g, A}$ and $Q_{n, B}$ of the order of 20 per cent will however, due to the logarithmic nature of expression (5) lead to an error of about $10 \mathrm{mV}$ in the determination of $\left|\phi_{s, A}-\phi_{s, B}\right|$ which is usually negligible.

\section{THE MEASUREMENT APPARATUS}

The principle of the measurement is shown in Fig. 7(a). A constant charging current $I$ is fed into the inverting input of an operational amplifier, connected to the bulk electrode of an MOS transistor. The bulk electrode has been shorted to the drain and source electrodes. The gate of the MOS transistor is connected to the output of the operational amplifier and an $x y$-recorder, on which $V_{g}$ is plotted vs. time.

As the rate of change of the charge flowing into the gate is constant the recording of $V_{g}$ vs. time will be equivalent to a recording of $V_{g}$ vs. the charge $Q_{g}$. 


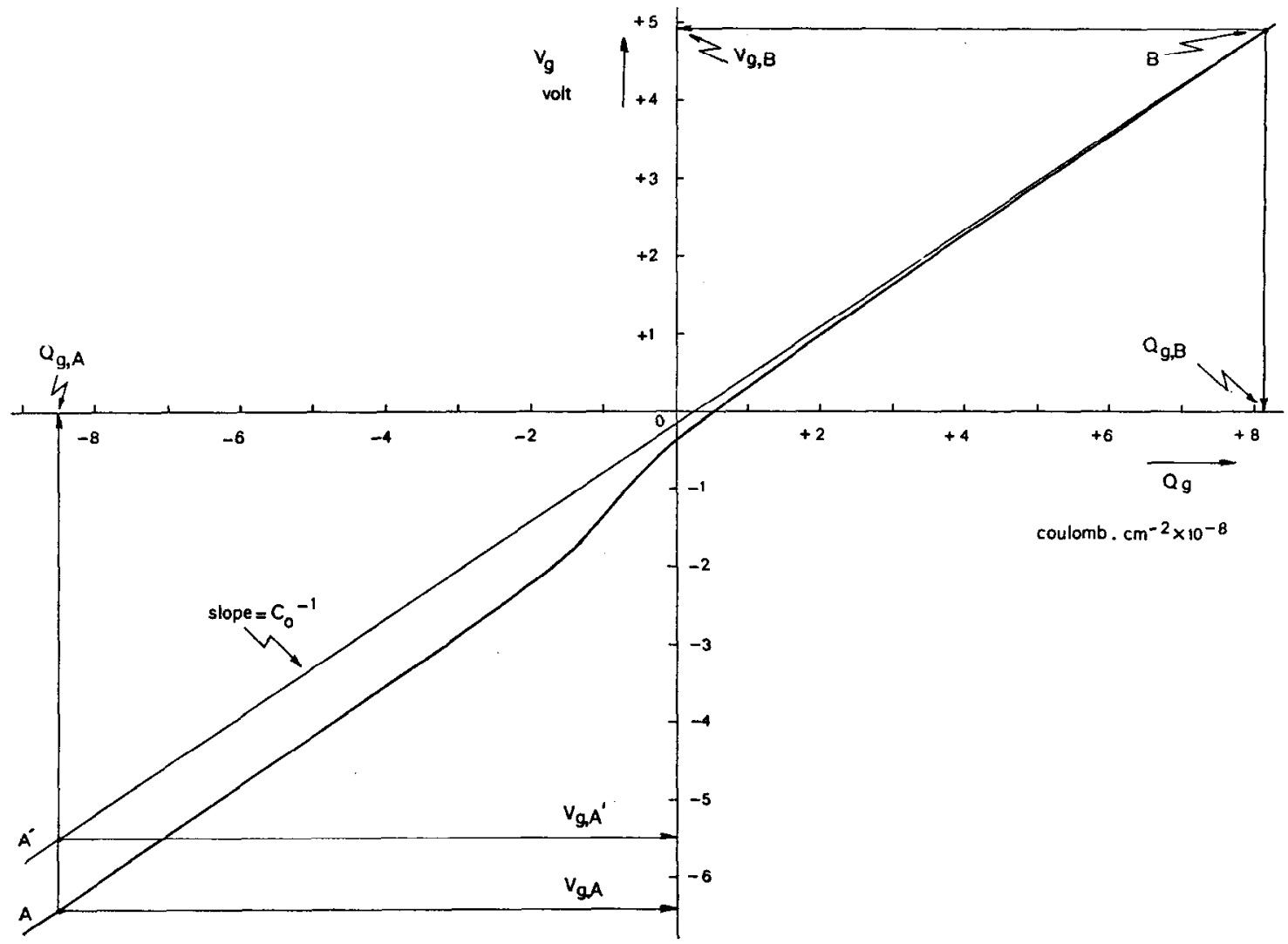

Fig. 3. The gate voltage $V_{g}$ as a function of the gate charge $Q_{g}$ for the same MOS transistor as in Fig. 2.

The battery in the gate circuit is needed to adjust the startpoint of the $V_{g}-Q_{g}$ plot.

In practice the current source $I$ and the operational amplifier in Fig. 7(a) are provided by the Keithley solid state electrometer model 602 , used in the 'Ohmmeter operation' condition. The

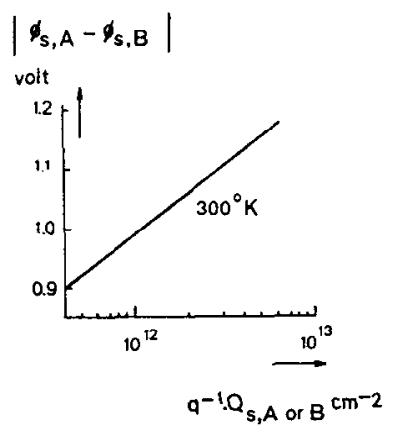

Fig. 4. The computed values $\left|\phi_{s, A}-\phi_{s, B}\right|$ as a function of $q^{-1} Q_{s, A}$ or $B$. electrometer is connected as illustrated in Fig. 7(b) It is used as an operational amplifier with terminals $\mathrm{J} 101$ and .1102 as the differential input. The terminal J106 acts as the inverting output and controls the gate voltage $V_{g}$ of the MOS transistor. The current source $I$ is provided within the

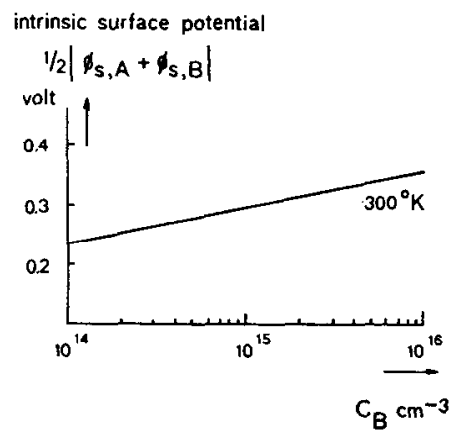

Fig. 5. The intrinsic surfacepotential $\frac{1}{2}\left|\phi_{s, A}+\phi_{s, B}\right|$ as a function of the bulk dope density $C_{B}$. 


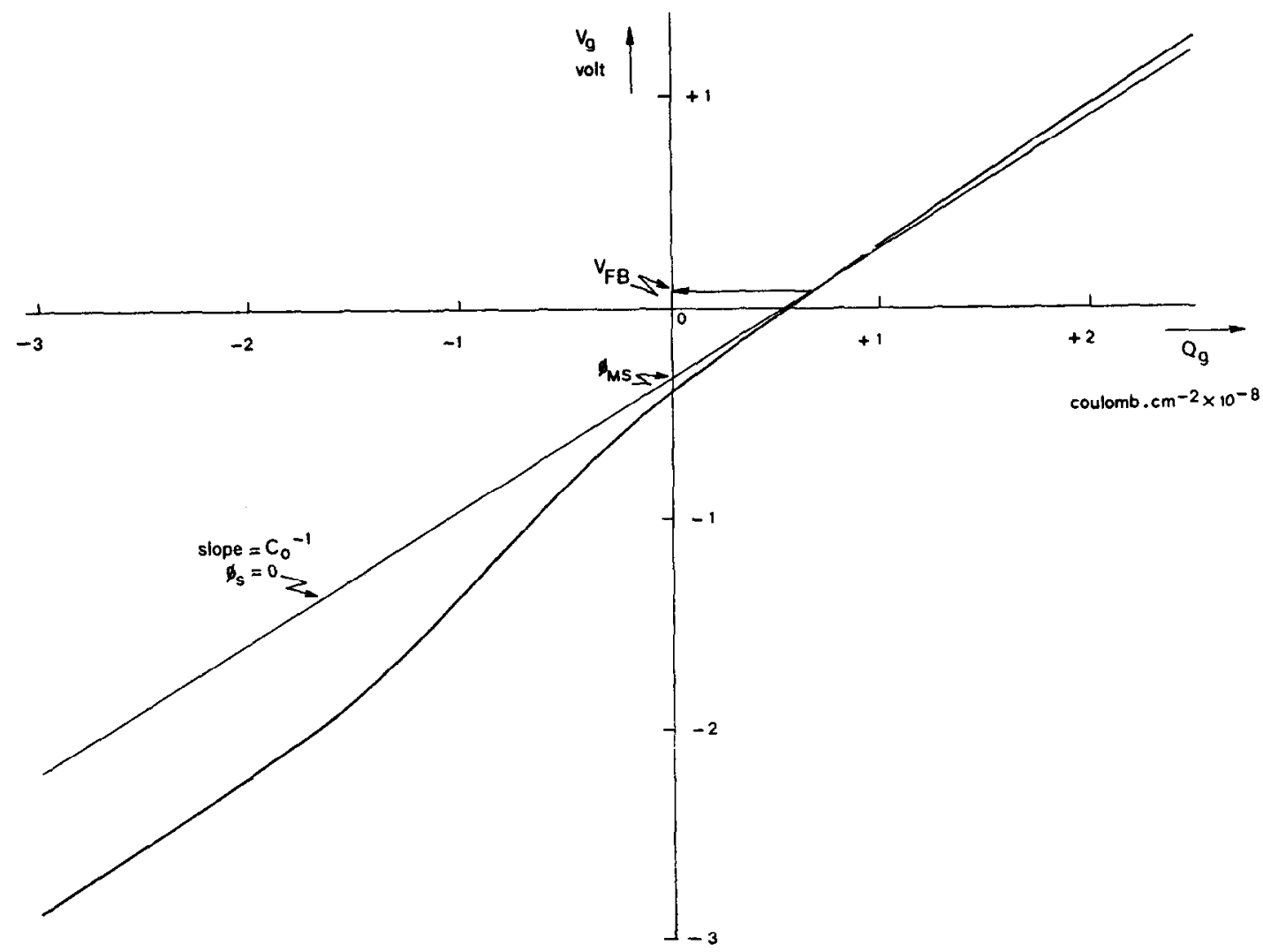

Fig. 6. The gate voltage $V_{\mathrm{g}}$ as a function of the gate charge $Q_{g}$ for the same MOS transistor as in Fig. 2.

electrometer between the terminals $\mathrm{J} 101$ and $\mathrm{J} 102$ and may be chosen between the values $10^{-12}$ and $10^{-5} \mathrm{~A}$. The zero switch allows short circuiting of the terminals $J 101$ and $J 102$. With the zero switch shorted the voltage supply $A$ can be adjusted for the desired startpoint of the $V_{g}-Q_{g}$ plot.

Typical values for the gate current density in the charge measuring method should lie between $10^{-10}-10^{-9} \mathrm{~A} \mathrm{~cm}^{-2} \mathrm{sec}^{-1}$.

In our measurements shown in Fig. 9 the current

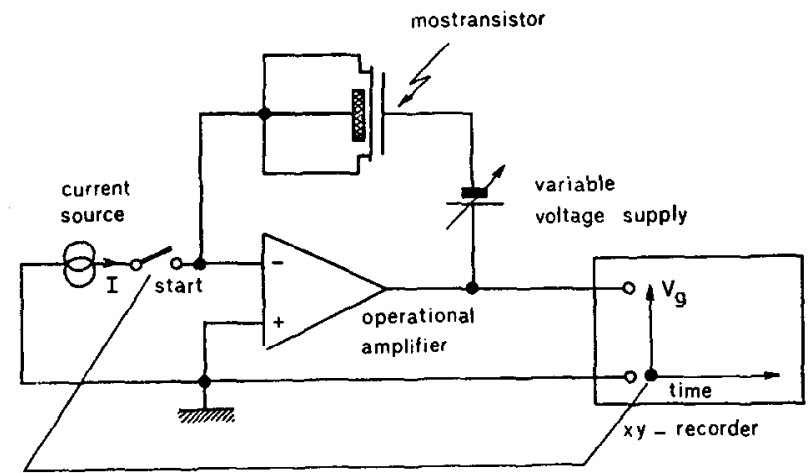

Fig. 7(a). Principle of the measurement. 


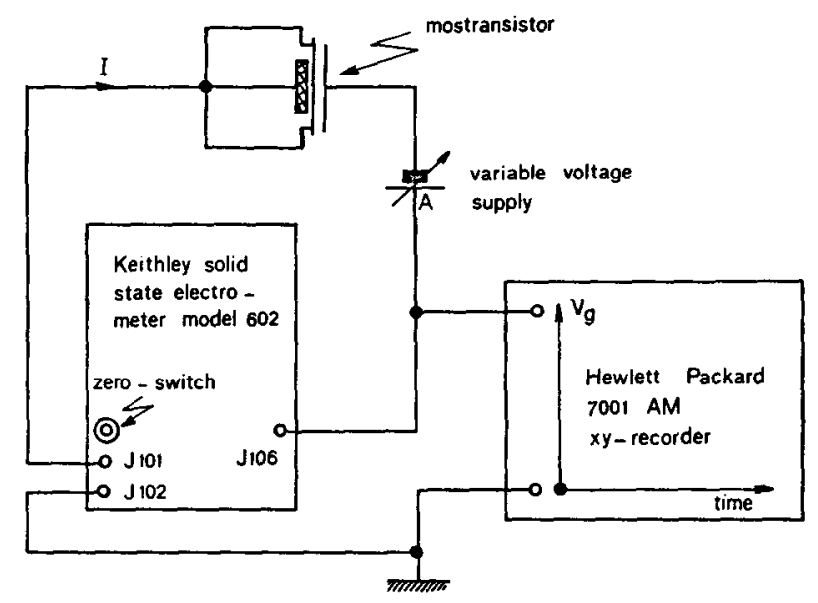

Fig. 7(b). The measurement apparatus.

source was set at $10^{-11} \mathrm{~A}$. The oxide capacitances $C_{0}$ of the samples 1,2 and 3 of Table 1 were 300 , 180 and $180 \mathrm{pF}$ respectively.

\section{ILLUSTRATION OF THE USE OF THE CHARGE MEASURING METHOD}

The method will be domonstrated on the following samples: an MOS transistor with relatively many surface states (sample 1) and two MOS transistors with relatively few surface states (the samples 2 and 3). The characteristics of the MOS transistors have been listed in Table 1. All the MOS transistors have the same geometry, represented in Fig. 8.

The total charge storage in the silicon and at the $\mathrm{Si}-\mathrm{SiO}_{2}$ interface $Q_{0}+Q_{s s}+Q_{s}$ of these samples has been graphically determined as a function of $\phi_{s}$ by the methods of the preceeding sections and the results are given in Figs. 9(a), (b) and (c). In

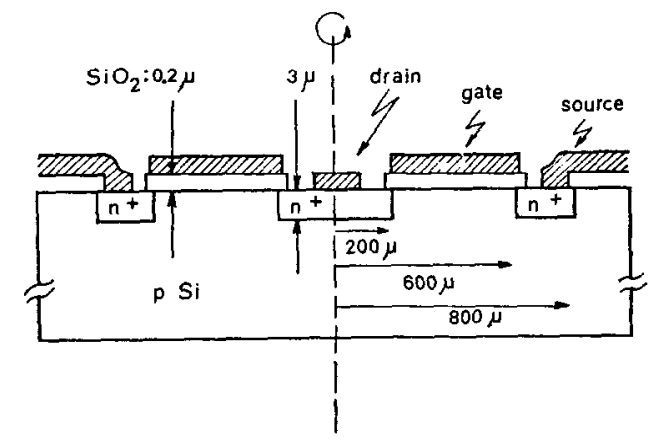

Fig. 8. Cross-section of the circular symmetrical MOS transistor used for the measurements. the same figures the charge $Q_{s}$ inside the silicon has also been plotted as a function of $\phi_{s} . Q_{s}\left(\phi_{s}\right)$ was obtained from curves published by Whelan [15].

The bulk dope $C_{B}$ has been determined by two independent measurements:

1. The measurement of the small signal capacitance between the bulk electrode and the shorted source and drain electrodes as a function of gatc bias, yielding the high frequency silicon space charge capacitance $C_{\mathrm{Si} \text {, min }}$ under the condition of inversion. The relation between $C_{\mathrm{Si} \text {, min }}$ and the bulk dope $C_{B}$ is given in [15].

2. A measurement of the influence of substrate bias upon the channel conduction of the MOS transistor, yielding the bulk dope $C_{B}$ in the condition of surface inversion [16].

The dope values determined by these methods have been presented in Table 1 and were found to be equal within experimental error. The value of the contact potential $\phi_{M S}$ has been taken from the literature [17].

The oxide capacitances $C_{0}$ obtained from the construction of Fig. 3 have been compared with the results of small signal capacitance measurements of $C_{0}(10 \mathrm{Khz})$ and were found to be equal. So there have been no serious leakage or polarization currents through the oxide during the charge measurements. By a subtraction of the charge inside the silicon $Q_{s}$ from the total charge $Q_{0}+Q_{s}$ । $Q_{s s}$, the charge storage in surface states $Q_{0}+Q_{s s}$ as a function of $\phi_{s}$ is determined and represented in Figs. 10(a), (b) and (c). 


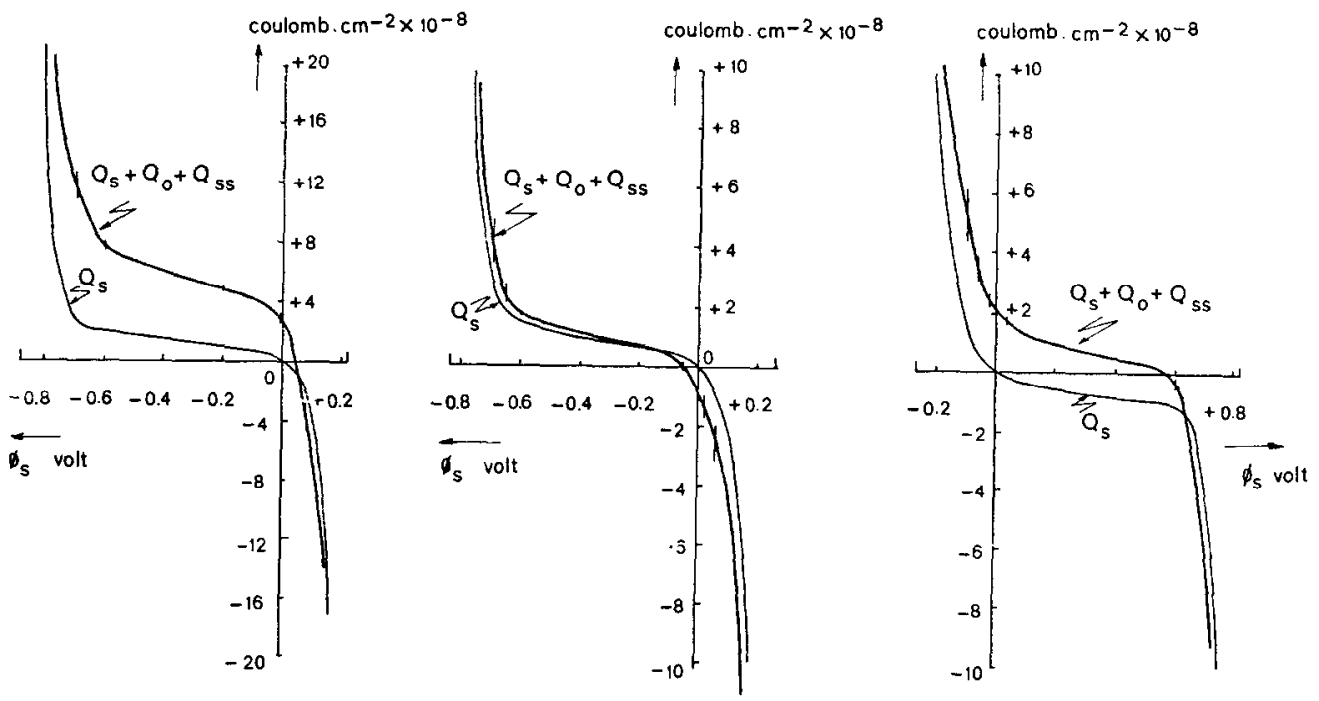

Fig. 9(a), (b) and (c). The charges $Q_{0}+Q_{s s}+Q_{s}$ and $Q_{s}$ as a function of $\phi_{s}$ for the samples 1,2 and 3 respectively.

Table 1. Characteristics of the MOS transistors used for the measurements

\begin{tabular}{|c|c|c|c|}
\hline Sample number: & 1 & 2 & 3 \\
\hline Type of silicon & $n$ & $n$ & $p$ \\
\hline Surface orientation & 1.1 .1$. & 1.0 .0 & 1.0 .0 \\
\hline \multicolumn{4}{|l|}{$\begin{array}{l}\text { Donor or acceptor } \\
\text { concentration of } \\
\text { bulk Si }\end{array}$} \\
\hline Determined by method I & $1.8 \times 10^{15}$ & $8 \times 10^{14}$ & $5.6 \times 10^{14}$ \\
\hline Determined by method 2 & $1.8 \times 10^{15}$ & $8 \times 10^{14}$ & $6.7 \times 10^{14}$ \\
\hline \multicolumn{4}{|l|}{ Thickness of the } \\
\hline insulator $(\AA)$ & 1200 & 2000 & 2000 \\
\hline \multicolumn{4}{|l|}{ Structure of the } \\
\hline insulator & $\mathrm{SiO}_{2}$ & \multirow{3}{*}{\multicolumn{2}{|c|}{$\begin{array}{l}\mathrm{SiO}_{2}-\mathrm{P}_{2} \mathrm{O}_{5} \\
\text { Oxide dry } \\
\text { grown at } \\
1200^{\circ} \mathrm{C}\end{array}$}} \\
\hline & Oxide dry & & \\
\hline & $\begin{array}{c}\text { grown at } \\
1150^{\circ} \mathrm{C}\end{array}$ & & \\
\hline & & \multicolumn{2}{|c|}{$\begin{array}{c}\text { Thickness of } \mathrm{P}_{2} \mathrm{O}_{5} \\
\text { glass layer: } \\
300 \text { à } 400 \AA\end{array}$} \\
\hline $\begin{array}{l}\text { Heat treatment } \\
\text { to remove surface } \\
\text { states }\end{array}$ & $\begin{array}{l}\text { No heat } \\
\text { treatment }\end{array}$ & \multicolumn{2}{|c|}{$\begin{array}{c}\text { Heat treatment: } \\
450^{\circ} \mathrm{C}-\mathrm{N}_{2} ; 80^{\circ} \mathrm{C}-\mathrm{H}_{2} \mathrm{O} \\
\text { during } 30^{\prime}\end{array}$} \\
\hline Gate metal & $\mathrm{Al}$ & Al & $\mathrm{Al}$ \\
\hline
\end{tabular}

*Samples 1 and 3 were manufactured at Philips Research Laboratories, Eindhoven, Netherlands and were obtained from $\mathrm{Mr}$. M. V. Whelan. Sample 2 was prepared in our laboratory by Mr. J. Holleman. 


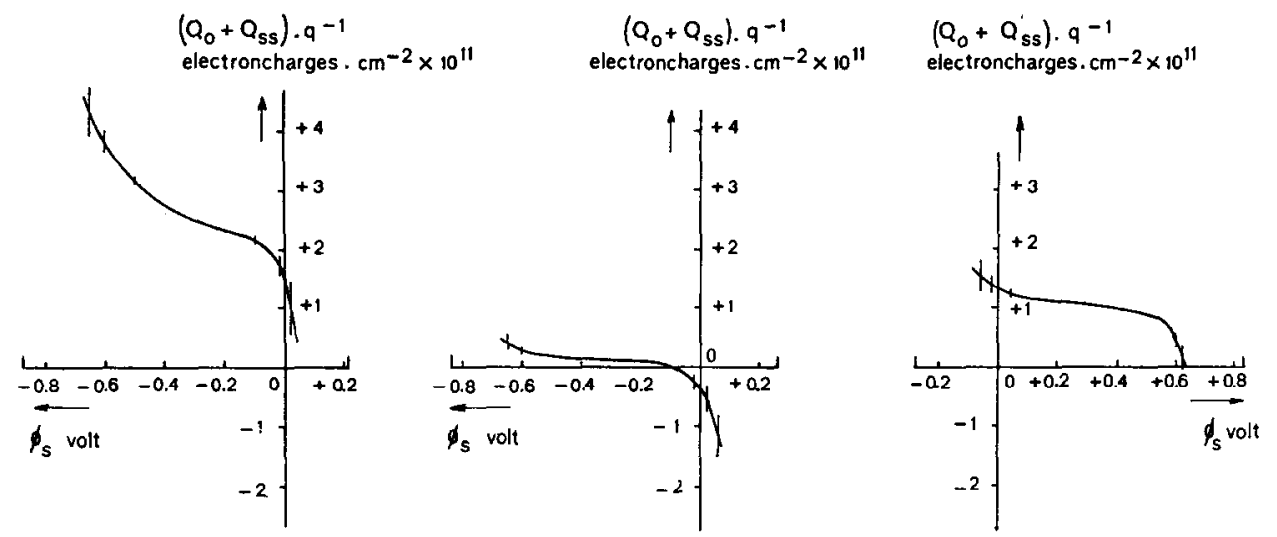

Fig. 10(a), (b) and (c). The charge $Q_{0}+Q_{s s}$ as a function of $\phi_{s}$ for the samples 1,2 and 3 respectively.

From Figs. 10(a), (b) and (c) we conclude that the rate of change of charge storage in surface states is relatively small for $\phi_{s}$ values in the intrinsic region of the silicon surface and relatively large for $\phi_{s}$ values in the regions near the valence band and conduction band edge of the surface of the semiconductor. This behaviour of charge storage in surface states has also been observed by other authors $[8,12,13]$ using different techniques for the investigation of the $\mathrm{Si}-\mathrm{SiO}_{2}$ interface.

The curves of Figs. 9 and 10 serve as examples of the charge measuring technique. In samples 2 and 3 different values of oxide charge $Q_{0}+Q_{s s}$ were found although the preparation of the samples was identical, according to Table 1 . Because the samples 2 and 3 were prepared at different laboratories (see footnote Table 1), we attribute these variations in $Q_{0}+Q_{s s}$ to other differing factors in the manufacturing processes by which samples 2 and 3 were obtained.

\section{DISCUSSION OF THE ERRORS ARISING IN THE APPLICATION OF THE CHARGE MEASURING METHOD}

An important condition for the interpretation of the $V_{g}-Q_{g}$ plot is the existence of thermal equilibrium inside the silicon material and at the $\mathrm{Si}-\mathrm{SiO}_{2}$ interface. Actually the $V_{g}-Q_{g}$ plot is measured under non-equilibrium conditions because during the measurement the charges in the silicon and at the $\mathrm{Si}-\mathrm{SiO}_{2}$ interface $Q_{0}+Q_{s s}+Q_{s}$ and in the gate $Q_{g}$ change with time. In general the shape of the $V_{g}-Q_{g}$ plot depends on the rate of change of gate charge $\mathrm{d} Q_{g} / \mathrm{d} t$ during the measurement of the $V_{g}-Q_{g}$ plot. However as found from the measure- ments for low values of $\mathrm{d} Q_{g} / \mathrm{d} t \quad\left(10^{-10}-10^{-9}\right.$ coulomb $\mathrm{cm}^{-2} \mathrm{sec}^{-1}$ ) the $V_{a}-Q_{o}$ plot appeared to be independent of this rate. Hence we conclude that the $V_{g}-Q_{g}$ plots have been measured under the condition of quasi-thermal equilibrium.

The estimated error in the determination of $C_{0}$ by the construction of Fig. 3 was less than 1 per cent. Also the error in the intrinsic surfacepotential

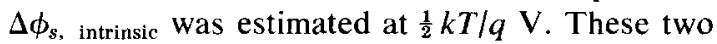
errors together determine the error $\Delta\left(Q_{0}+Q_{s s}+\right.$ $\left.Q_{s}\right)$ in the $Q_{0}+Q_{s s}+Q_{s}$ vs. $\phi_{s}$ plots of Figs. 9(a), (b) and (c). For the error $\Delta\left(Q_{0}+Q_{s s}+Q_{s}\right)$ as a function of $\phi_{s}$ the following expressions can be derived:

$$
\left|\Delta\left(Q_{0}+Q_{s s}+Q_{s}\right)\right|=\frac{\left|\Delta Q_{g}\right|}{\Delta \phi_{s}} \cdot \Delta \phi_{s}
$$

where

$$
\begin{array}{r}
\left|\Delta \phi_{s}\right|<\left|\Delta \phi_{s, \text { intrinsic }}+\frac{\Delta C_{0}}{C_{0}} \frac{\left|Q_{g}-Q_{g, \text { intrinsic }}\right|}{C_{0}}\right| \text { and } \\
\frac{\left|\Delta Q_{g}\right|}{\Delta \phi_{s}}=\frac{\Delta Q_{g}}{\Delta V_{g}} \frac{1}{\left|1-\left(\Delta Q_{g} / C_{0} \Delta V_{g}\right)\right|}
\end{array}
$$

The maximum error $\Delta\left(Q_{0}+Q_{s s}+Q_{s}\right)$ has been computed by applying (8) to various points of the curves of Figs. 9(a), (b) and (c) and its magnitude has been indicated by small vertical lines in Figs. 9(a), (b) and (c).

The error $\Delta\left(Q_{0}+Q_{s s}\right)$ in the $Q_{0}+Q_{s s}$ vs. $\phi_{s}$ plots of Fig. 10 is determined by the error in the surfacepotential $\Delta \phi_{s}$ mentioned before and the error in the determination of the bulk dope density $C_{B}$ (20 per cent). This maximum error $\Delta\left(Q_{0}+Q_{s s}\right)$ is estimated for various points of the curves of 
Figs. 10(a), (b) and (c) and its estimated magnitude has also been indicated by small vertical lines in Figs. 10(a), (b) and (c).

\section{COMPARISON OF THE CHARGE MEASURING METHOD WITH OTHER TECHNIQUES FOR INVESTIGATING THE $\mathrm{Si}_{-} \mathrm{SiO}_{2}$ INTERFACE}

The charge measuring method is an alternative method for the investigation of the $\mathrm{Si}-\mathrm{SiO}_{2}$ interface. This method resembles in a way the low frequency thermal equilibrium $C-V$ measurement method of Berglund [9]. Therefore we shall compare these two methods first.

Both methods yield the surface charge as a function of surfacepotential; in Berglund's method from an analysis of the derivative of the surface charge vs. surfacepotential $\mathrm{d}\left(Q_{s s}+Q_{s}\right) / \mathrm{d} \phi_{s}$ and in the charge measuring method from a direct analysis of the $V_{g}-Q_{g}$ plot. One of the advantages of the charge measuring method is that information about the fixed oxide charge $Q_{0}$ is not 'lost'. On the other hand Berglund's method will be more sensitive for a variation of surface state charge density as a function of surfacepotential as it measures incremental charge rather than total charge.

In both methods the relationship between the measured gate voltage $V_{g}$ and the actual surfacepotential $\phi_{s}$ should be established. In the $C-V$ method an integration step is required and in the charge measuring method the relationship can be read directly from the $V_{g}-Q_{g}$ plot. Also in both methods a 'matchpoint' is needed to link quantitatively the surfacepotential $\phi_{s}$ and the voltage $V_{g}$. We are of the opinion that the use of the intrinsic condition as a matchpoint has advantages over the use of the accumulation condition as a matchpoint, as done by Berglund. In the $C-V$ method, especially if this method is employed on MOS transistors, the procedure for finding the intrinsic point may be based upon the procedure applied in the charge measuring method that we hope to outline in a forthcoming publication.

It should be admitted that the $V_{g}-Q_{g}$ plot can also be obtained by electronically integrating a very low frequency $C-V$ plot. We have not looked into this possibility, which in principle yields the same information, but which requires more complex instrumentation.

When we compare the charge measuring method with the high frequency $C-V$ measurement method we notice similar differences as with the low frequency $C-V$ method due to the incremental nature of $C-V$ measurements.

Furthermore the range of surfacepotentials attainable with the charge measuring method will generally be larger and the influence of bulk series resistance will be negligible.

In conclusion we believe that the measurement of gate voltage vs. gate charge constitutes a lucid and easy-to-instrument method to obtain information about charge at the $\mathrm{Si}-\mathrm{SiO}_{2}$ interface.

Acknowledgements-Grateful acknowledgèments are due to Prof. Ir. O. W. Memelink for helpful discussions.

\section{REFERENCES}

1. C. G. B. Garrett and W. H. Brattain, Phys. Rev. 99 , 376, (1955).

2. R. Lindner, Bell Syst. tech.J. 41, 803, (1962).

3. L. M. Terman, Solid-St. Electron. 5, 285, (1962).

4. K. Lehovec, A. Slobodskoy and J. L. Sprague, Phys. Stat. Sol 3, 447, (1963).

5. F. P. Heiman and G. Warfield, IEEE Trans. Electron Devices, ED-12, 167, (1965).

6. K. H. Zaininger and G. Warficld, IEEE Trans. Electron Devices, ED-12, 179, (1965).

7. A. S. Grove, B. E. Deal, E. H. Snow and C. T. Sah, Solid-St. Electron. 8, 145. (1965).

8. M. V. Whelan, Philips Res. Rep. 20, 562, (1965).

9. C. N. Berglund, IEEE Trans. Electron Devices, ED-13, 701, (1966).

10. P. V. Gray and D. M. Brown, App. Phys. Letters, 8 , $31,(1966)$.

11. E. H. Nicollian and A. Goetzberger, Appl Phys. Letters, 10, 60, (1967).

12. E. H. Nicollian and A. Goetzberger, Bell Syst. tech. J. 46, 1055, (1967).

13. E. Arnold, IEEE Trans. Electron Devices, ED-15, 1003, (1968).

14. M. Kuhn, Recent News Paper, Electrochem. Soc: Conf., New York, May (1969).

15. M. V. Whelan, Philips Res. Rep. 20, 620-632, (1965).

16. J. A. van Nielen and O. W. Memelink, Philips Res. Rep. 22, 55-71, (1967).

17. A. S. Grove, Physics and Technology of Semiconductor Devices, John Wiley, New York, (1967).

18. D. R. Kerr, M.I.S. Measurement techniques utilizing slow voltage ramps, Presented at the conference on Properties and use of M.I.S. structures, Grenoble, France. 17-20 June (1969). 